\title{
Characteristics and prognostic factors of colorectal mucinous adenocarcinoma with signet ring cells
}

This article was published in the following Dove Press journal:

Cancer Management and Research

2 November 2017

Number of times this article has been viewed

\author{
Xiangquan Kong* \\ Xueqing Zhang* \\ Yunxia Huang \\ Lirui Tang \\ Qingqin Peng \\ Jinluan Li
}

Department of Radiation Oncology, Fujian Medical University Cancer Hospital, Fujian Cancer Hospital, Fuzhou, People's Republic of China

*These authors contributed equally to this work.
Correspondence: Jinluan $\mathrm{Li}$ Department of Radiation Oncology, Fujian Medical University Cancer Hospital, Fujian Cancer Hospital, 420 Fuma Road, Jin'an District, Fuzhou 3500 I4, People's Republic of China Email lijinluan@pku.org.cn
Background: Colorectal signet ring cell (SRC) carcinoma occurs rarely with a poor prognosis. The present study assessed the prognostic factors and predictive value of SRC ratio in colorectal mucinous adenocarcinoma (MAC) with SRCs (MAC-SRC).

Patients and methods: A total of 95 consecutive colorectal MAC-SRC patients, confirmed pathologically from February 1987 to December 2015, were analyzed retrospectively in our institute. Clinical characteristics, pathological grade, TNM staging, and SRC ratio were assessed to identify the prognostic factors related to progression-free survival (PFS) and overall survival (OS). SPSS 22.0 was used for statistical analyses.

Results: The median follow-up time was 29.7 months (range 0.8-165). Meanwhile, 5-year PFS and OS rates were $25.6 \%$ (95\% confidence interval [CI] 16.192-35.008\%) and 40.5\% (95\% CI 29.524-51.476\%), respectively. Among the 81 patients who underwent surgery, 78 (96.3\%) were diagnosed as stage T3 or T4; 74 (91.4\%) showed lymph node involvement, and $27(29.3 \%)$ presented distant metastasis. Metastases of the peritoneal cavity and ovaries were observed commonly in colorectal MAC-SRC. In the multivariate Cox regression model, SRC ratio $\geq 35 \%$, absence of preoperative radiotherapy, and distant metastasis were independent predictors of PFS. Furthermore, SRC ratio $\geq 35 \%$, absence of preoperative chemotherapy (pre$\mathrm{CT}$ ), and distant metastasis were independent risk factors for poor prognosis.

Conclusion: A long-term follow-up of colorectal MAC-SRC reveals that it is a rare subtype of colorectal MAC with a dismal prognosis. Furthermore, SRC ratio, pre-CT, and M stage seem to affect OS independently.

Keywords: colorectal mucinous adenocarcinoma, signet ring cell, progression-free survival, overall survival, prognostic factors

\section{Introduction}

Mucinous adenocarcinoma (MAC) is an epithelial malignancy characterized by distinctive cells secreting abundant extracellular mucin. ${ }^{1} \mathrm{SRCC}$ is a rare subtype of MAC with ringlike cells in which the nucleus is displaced to the periphery by substantial intracytoplasmic mucin. ${ }^{2}$ Verhulst et $\mathrm{al}^{3}$ reported that colorectal SRCC is rare, and accounts for $<1 \%$ of all colorectal adenocarcinoma cases. Colorectal SRCC is associated with younger age, higher tumor grade, and poorer outcomes, compared with conventional adenocarcinoma. ${ }^{4-9}$

The World Health Organization defines SRCC as an adenocarcinoma with $\geq 50 \% \mathrm{SRCs}$ in the tumor. ${ }^{10}$ However, whether prognosis is associated with the SRC ratio in colorectal MAC-SRC remains unclear. Indeed, colorectal MAC comprising low SRC amounts $(<50 \%)$ has been seldom evaluated previously. Therefore, it is essential to assess the 
characteristics and prognostic factors of colorectal MAC-SRC in order to improve early diagnosis, treatment, and surveillance.

In the current study, we retrospectively analyzed the long-term outcomes of 95 patients with colorectal MAC$\mathrm{SRC}$ in our institute, and evaluated the predictive value of the SRC ratio.

\section{Patients and methods}

\section{Patients}

A total of 95 consecutive colorectal MAC-SRC patients, from February 1987 to December 2015, were examined retrospectively. They had (a) colorectal MAC-SRC, confirmed pathologically by 2 pathologists independently through H\&E staining in a retrospective review; (b) complete medical records with respect to demographic characteristics and laboratory findings; (c) no previous or concurrent malignancy; and (d) follow-up for more than 6 months after the definite diagnosis. Disease staging was performed according to the AJCC, 8th edition. ${ }^{11}$ The present study was approved by the Ethics Committee of Fujian Cancer Hospital, Fuzhou, People's Republic of China (KT2017-010). Patient medical records and tumor specimens were analyzed retrospectively, with no individual patient identifiable information used. Therefore, individual consent was not required by the Ethics Committee.

\section{Follow-up}

The patients were followed up clinically every 3 months for 1 year, every 6 months for the subsequent 2 years, and annually thereafter. Tumor progression was defined either pathologically or radiologically as local recurrence (recurrence close to the primary tumor bed or at the anastomotic stoma) and distant metastases (relapses in regions that could not be regarded as local recurrence).

\section{Statistical analysis}

Research end points were PFS and OS. The Kaplan-Meier method was used to generate survival curves, with the logrank test employed for comparison. Meanwhile, the cutoff point for the SRC ratio affecting survival was calculated using Cutoff Finder application. ${ }^{12}$ A Cox regression model was used to evaluate predictive factors of PFS and OS. Two-sided $P<0.05$ was considered statistically significant. PFS was defined as the time from pathological diagnosis to tumor progression or death. OS was determined as the time interval between pathological diagnosis and death due to any reason or last follow-up. Statistical analyses were conducted with SPSS version 22.0 (IBM Corporation, Armonk, NY, USA).

\section{Results}

\section{Patient characteristics}

A total of 95 patients were enrolled in the present study. Table 1 shows their baseline characteristics. Median age was 44 years (range 22-82), and the cohort included 47 $(49.5 \%)$ males and 48 (50.5\%) females. Among the 95 cases, $62.1 \%(59 / 95)$ had primary lesions in the rectum. Of the included patients, 52.1\% (37/71) exhibited high pre-

Table I Patient characteristics

\begin{tabular}{|c|c|c|}
\hline Characteristics & & Data, n (\%) \\
\hline \multicolumn{3}{|l|}{ Gender } \\
\hline & Male & $47(49.5)$ \\
\hline & Female & $48(50.5)$ \\
\hline \multicolumn{3}{|l|}{ Age } \\
\hline & Median (range) & $44(22-82)$ \\
\hline & $\leq 40$ & $38(40.0)$ \\
\hline & $>40$ & $57(60.0)$ \\
\hline \multicolumn{3}{|l|}{ Location } \\
\hline & Ileocecal junction & $7(7.4)$ \\
\hline & Right colon & $12(12.6)$ \\
\hline & Left colon & $17(17.9)$ \\
\hline & Rectum & $59(62.1)$ \\
\hline \multicolumn{3}{|l|}{ SRC ratio } \\
\hline & $<35$ & $38(40.0)$ \\
\hline & $\geq 35$ & $57(60.0)$ \\
\hline \multicolumn{3}{|l|}{ Pre-CEA (ng/mL) } \\
\hline & $<5.0$ & $34(35.8)$ \\
\hline & $\geq 5.0$ & $37(38.9)$ \\
\hline & Unknown & $24(25.3)$ \\
\hline \multicolumn{3}{|l|}{ Bowel obstruction } \\
\hline & Yes & $21(22.1)$ \\
\hline & No & 74 (77.9) \\
\hline \multicolumn{3}{|l|}{ Pre-CT } \\
\hline & Yes & $8(8.4)$ \\
\hline & No & $87(91.6)$ \\
\hline \multicolumn{3}{|l|}{ Pre-RT } \\
\hline & Yes & $9(9.5)$ \\
\hline & No & $86(90.5)$ \\
\hline \multicolumn{3}{|l|}{ T stage } \\
\hline & I & $\mathrm{I}(\mathrm{II})$ \\
\hline & 2 & $2(2.1)$ \\
\hline & 3 & $43(45.3)$ \\
\hline & 4 & $35(36.8)$ \\
\hline & Unknown & $14(14.7)$ \\
\hline \multicolumn{3}{|l|}{$\mathrm{N}$ stage } \\
\hline & 0 & $7(7.4)$ \\
\hline & 1 & $16(16.8)$ \\
\hline & 2 & $58(61.1)$ \\
\hline & Unknown & $14(14.7)$ \\
\hline \multicolumn{3}{|l|}{ M stage } \\
\hline & 0 & $65(68.4)$ \\
\hline & 1 & $27(28.4)$ \\
\hline & Unknown & $3(3.2)$ \\
\hline
\end{tabular}

Abbreviations: SRC, signet ring cell; pre-CEA, preoperative carcinoembryonic antigen; pre-CT, preoperative chemotherapy; pre-RT, preoperative radiotherapy. 
CEA levels ( $\geq 5.0 \mathrm{ng} / \mathrm{mL})$, while $47.9 \%(34 / 71)$ had normal amounts $(<5.0 \mathrm{ng} / \mathrm{mL})$. In addition, $8(8.4 \%)$ and $9(9.5 \%)$ patients underwent pre-CT and pre-RT, respectively. Fortyeight $(50.5 \%)$ patients underwent post-CT, and $16(16.8 \%)$ underwent post-RT.

\section{Pathological features}

According to the Cutoff Finder software, 35\% was considered the optimal cutoff point for the SRC ratio (Figure 1). In all, $38(40.0 \%)$ of 95 patients had $<35 \%$ SRCs, while $57(60 \%)$ had values $\geq 35 \%$. In this population, 81 patients underwent surgery; among them, 78 (96.3\%) were diagnosed as stage T3 or T4, with 74 (91.4\%) showing lymph node involvement and $27(33.3 \%)$ presenting distant metastasis. Twelve (14.8\%), $35(43.2 \%)$, and $16(19.8 \%)$ patients showed tumor nodules, LVI, and neural invasion, respectively.

\section{Local control and survival outcomes}

The median follow-up time was 29.7 months (range 0.8-165). In the current study, 84 of $95(88.4 \%)$ patients experienced disease progression, of which 26/84 (40.0\%) and 25/84 (29.8\%) had locoregional relapse and distant metastasis, respectively. Locoregional relapse cases were of presacral $(4 / 26,15.4 \%)$, anastomotic $(7 / 26,26.9 \%)$, and pelvic $(17 / 26$, $65.4 \%)$ types. Common distant metastasis sites included the peritoneum $(8 \%, 2 / 25)$, ovary $(12.0 \%, 3 / 25)$, lung $(16.0 \%$, $4 / 25)$, bone $(28.0 \%, 7 / 25)$, and lymph nodes $(40.0 \%, 10 / 25)$. Median PFS was 22.9 months (range 0.7-164.9); 1-, 3-, and 5-year PFS rates for the whole population were $67.0 \%(95 \%$ CI 57.396-76.604\%), 42.3\% (95\% CI 32.108-52.492\%), and $25.6 \%$ (95\% CI 16.192-35.008\%), respectively. Meanwhile, 1-, 3-, and 5-year OS rates were 79.3\% (95\% CI 71.068-87.532\%), 52.3\% (95\% CI 41.716-62.884\%), and $42.5 \%$ (95\% CI 31.720-53.280\%), respectively.

\section{Prognostic factors of PFS and OS}

Table 2 summarizes the univariate and multivariate analyses of factors affecting PFS. Univariate analysis indicated that SRC ratio, bowel obstruction, $M$ stage, and LVI were significantly associated with PFS (all $P<0.05$ ). In addition,
A

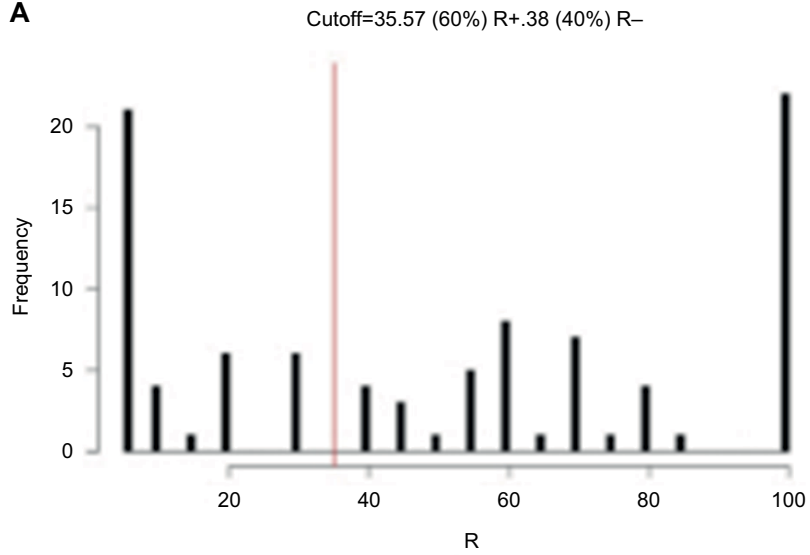

C

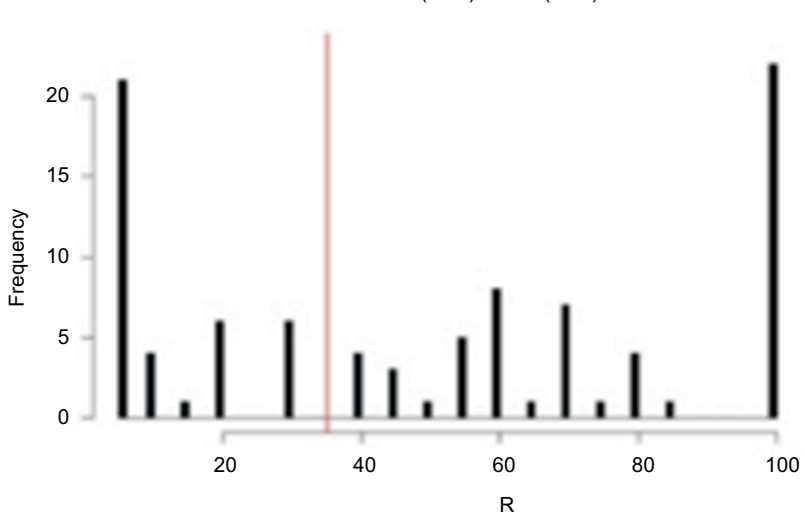

B

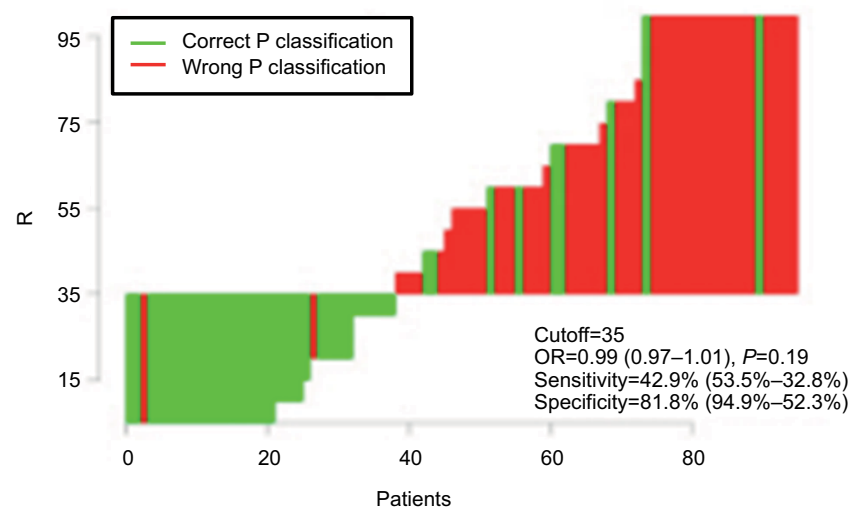

D

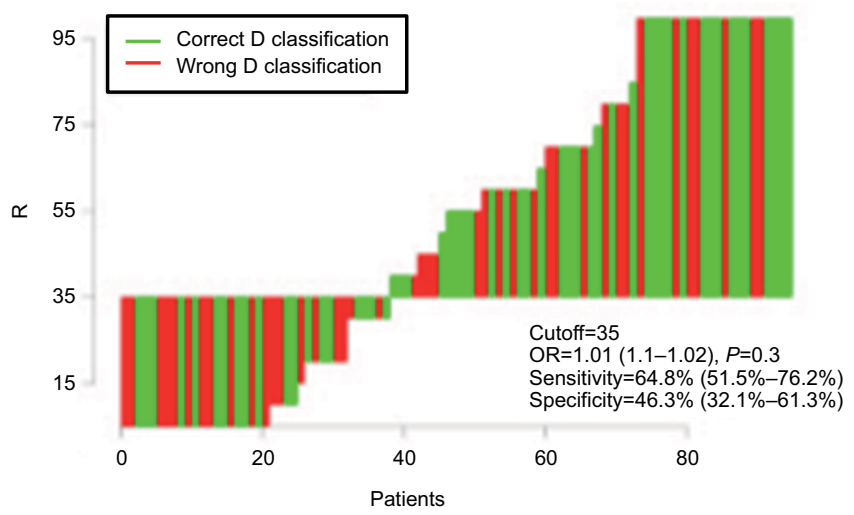

Figure I Distribution-based cutoff optimization in colorectal MAC-SRC patients. (A and C) Histograms of SRC ratios in 95 colorectal MAC-SRC cases. The vertical red line indicates the optimal cutoffs derived from the survival-based model. (B and D) Waterfall plot of optimal dichotomization. Classification was done using the SRC ratio status; optimal cutoff was assessed for the events of progression and death.

Abbreviations: R, ratio; P, progression; D, death; OR, odds ratio; MAC-SRC, mucinous adenocarcinoma with SRCs; SRC, signet ring cell. 
Table 2 Correlation between clinicopathological factors and progression-free survival

\begin{tabular}{|c|c|c|c|c|}
\hline \multirow[t]{2}{*}{ Characteristics } & \multicolumn{2}{|c|}{ Univariate } & \multicolumn{2}{|l|}{ Multivariate } \\
\hline & $\chi^{2}$ & $P$ & HR (95\% Cl) & $P$ \\
\hline \multicolumn{5}{|l|}{ Gender } \\
\hline Male/female & 1.648 & 0.199 & & \\
\hline \multicolumn{5}{|l|}{ Age } \\
\hline$\leq 40 />40$ & 0.026 & 0.871 & & \\
\hline \multicolumn{5}{|l|}{ Location } \\
\hline $\begin{array}{l}\text { Ileocecal junction/ } \\
\text { right colon/left colon/ } \\
\text { rectum }\end{array}$ & 4.949 & 0.176 & & \\
\hline \multicolumn{5}{|l|}{ SRC ratio } \\
\hline$<35 / \geq 35$ & 3.915 & 0.048 & $1.917(1.140-3.225)$ & 0.014 \\
\hline \multicolumn{5}{|l|}{ Pre-CEA (ng/mL) } \\
\hline$<5.0 / \geq 5.0$ & 2.895 & 0.089 & & \\
\hline \multicolumn{5}{|l|}{ Bowel obstruction } \\
\hline Yes/no & 3.918 & 0.048 & 1.633 (0.892-2.99| & 0.112 \\
\hline \multicolumn{5}{|l|}{ Pre-CT } \\
\hline Yes/no & 2.003 & 0.157 & & \\
\hline \multicolumn{5}{|l|}{ Pre-RT } \\
\hline Yes/no & 3.752 & 0.053 & 3.394 (I.27I-9.060) & 0.015 \\
\hline \multicolumn{5}{|l|}{ T stage } \\
\hline $1 / 2 / 3 / 4$ & 3.968 & 0.265 & & \\
\hline \multicolumn{5}{|l|}{$\mathrm{N}$ stage } \\
\hline $0 / 1 / 2$ & 4.733 & 0.094 & & \\
\hline \multicolumn{5}{|l|}{ M stage } \\
\hline $0 / 1$ & 6.959 & 0.008 & $1.750(1.015-3.017)$ & 0.044 \\
\hline \multicolumn{5}{|l|}{ Carcinoma nodules } \\
\hline \pm & 0.389 & 0.533 & & \\
\hline \multicolumn{5}{|l|}{ Lymphovascular invasion } \\
\hline \pm & 4.584 & 0.032 & $1.662(0.972-2.843)$ & 0.063 \\
\hline \multicolumn{5}{|l|}{ Neural invasion } \\
\hline \pm & 1.191 & 0.275 & & \\
\hline \multicolumn{5}{|l|}{ Post-CT } \\
\hline Yes/no & 0.663 & 0.416 & & \\
\hline \multicolumn{5}{|l|}{ Post-RT } \\
\hline Yes/no & 0.889 & 0.346 & & \\
\hline
\end{tabular}

Abbreviations: $\mathrm{HR}$, hazard ratio; $\mathrm{Cl}$, confidence interval; $\mathrm{SRC}$, signet ring cell; preCEA, preoperative carcinoembryonic antigen; pre-CT, preoperative chemotherapy; pre-RT, preoperative radiotherapy; post-CT, postoperative chemotherapy; post$\mathrm{RT}$, postoperative radiotherapy.

pre-RT was almost significantly correlated to PFS $(P=0.053)$. Of these variables, SRC ratio $(P=0.014, \mathrm{HR}=1.917,95 \%$ CI 1.140-3.225), pre-RT $(P=0.015, \mathrm{HR}=3.394,95 \% \mathrm{CI}$ $1.271-9.060)$, and $\mathrm{M}$ stage $(P=0.044, \mathrm{HR}=1.750,95 \% \mathrm{CI}$ 1.015-3.017) were independent prognostic factors of PFS. Univariate and multivariate analyses of OS are shown in Table 3. Univariate analysis revealed that SRC ratio, pre$\mathrm{CEA}$, pre-CT, and $\mathrm{M}$ stage were significantly associated with OS (all $P<0.05$ ). Meanwhile, multivariate analysis showed that $\mathrm{SRC}$ ratio $(P=0.032, \mathrm{HR}=2.500,95 \% \mathrm{CI} 1.082-5.781)$, pre-CT $(P=0.031, \mathrm{HR}=3.755,95 \% \mathrm{CI} 1.127-12.517)$, and M stage $(P=0.000, \mathrm{HR}=3.219,95 \%$ CI $1.676-6.181)$ were independent predictive factors of OS. Figure 2 displays the
Table 3. Correlation between clinicopathological factors and overall survival (OS).

\begin{tabular}{|c|c|c|c|c|}
\hline \multirow[t]{2}{*}{ Characteristics } & \multicolumn{2}{|c|}{ Univariate } & \multicolumn{2}{|l|}{ Multivariate } \\
\hline & $\chi^{2}$ & $P$ & HR (95\% Cl) & $P$ \\
\hline \multicolumn{5}{|l|}{ Gender } \\
\hline Male/female & 2.317 & 0.128 & & \\
\hline \multicolumn{5}{|l|}{ Age } \\
\hline$\leq 40 />40$ & 0.003 & 0.959 & & \\
\hline \multicolumn{5}{|l|}{ Location } \\
\hline $\begin{array}{l}\text { Ileocecal junction/ } \\
\text { right colon/left } \\
\text { colon/rectum }\end{array}$ & $1.81 \mathrm{I}$ & 0.613 & & \\
\hline \multicolumn{5}{|l|}{ SRC ratio } \\
\hline$<35 / \geq 35$ & 4.469 & 0.035 & $2.500(\mathrm{I} .082-5.78 \mathrm{I})$ & 0.032 \\
\hline \multicolumn{5}{|l|}{ Pre-CEA (ng/mL) } \\
\hline$<5.0 / \geq 5.0$ & 8.423 & 0.004 & $1.808(0.893-3.660)$ & 0.100 \\
\hline \multicolumn{5}{|l|}{ Bowel obstruction } \\
\hline Yes/no & 0.521 & 0.470 & & \\
\hline \multicolumn{5}{|l|}{ Pre-CT } \\
\hline Yes/no & 4.505 & 0.034 & $3.755(1.127-12.517)$ & 0.031 \\
\hline \multicolumn{5}{|l|}{ Pre-RT } \\
\hline Yes/no & 2.964 & 0.085 & & \\
\hline \multicolumn{5}{|l|}{ T stage } \\
\hline $1 / 2 / 3 / 4$ & 2.993 & 0.393 & & \\
\hline \multicolumn{5}{|l|}{$\mathrm{N}$ stage } \\
\hline $0 / 1 / 2$ & 4.969 & 0.083 & & \\
\hline \multicolumn{5}{|l|}{ M stage } \\
\hline $0 / 1$ & $13.17 \mid$ & 0.000 & $3.219(1.676-6.181)$ & 0.000 \\
\hline \multicolumn{5}{|l|}{ Carcinoma nodules } \\
\hline \pm & 3.137 & 0.077 & & \\
\hline \multicolumn{5}{|l|}{ Lymphovascular } \\
\hline \multicolumn{5}{|l|}{ invasion } \\
\hline \pm & 2.108 & 0.147 & & \\
\hline \multicolumn{5}{|l|}{ Neural invasion } \\
\hline \pm & 0.698 & 0.403 & & \\
\hline \multicolumn{5}{|l|}{ Post-CT } \\
\hline Yes/no & 0.207 & 0.649 & & \\
\hline \multicolumn{5}{|l|}{ Post-RT } \\
\hline Yes/no & 1.397 & 0.237 & & \\
\hline
\end{tabular}

Abbreviations: $\mathrm{HR}$, hazard ratio; $\mathrm{Cl}$, confidence interval; SRC, signet ring cell; preCEA, preoperative carcinoembryonic antigen; pre-CT, preoperative chemotherapy; pre-RT, preoperative radiotherapy; post-CT, postoperative chemotherapy; post$\mathrm{RT}$, postoperative radiotherapy.

impact of the SRC ratio ( $<35 \mathrm{vs} \geq 35 \%$ ) on survival according to the Kaplan-Meier analysis.

\section{Discussion}

This is one of the few studies focusing on the predictive value of SRC ratio in colorectal MAC-SRC patients with multiple prognostic factors. Our findings showed that SRC ratio $\geq 35 \%$ and distant metastasis indicated poor prognosis in colorectal MAC-SRC, while pre-CT and pre-RT improved survival.

Colorectal MAC has a dismal prognosis compared to classical adenocarcinoma. ${ }^{1,13}$ The reported 5-year OS rate is significantly lower for colorectal MAC than classical 

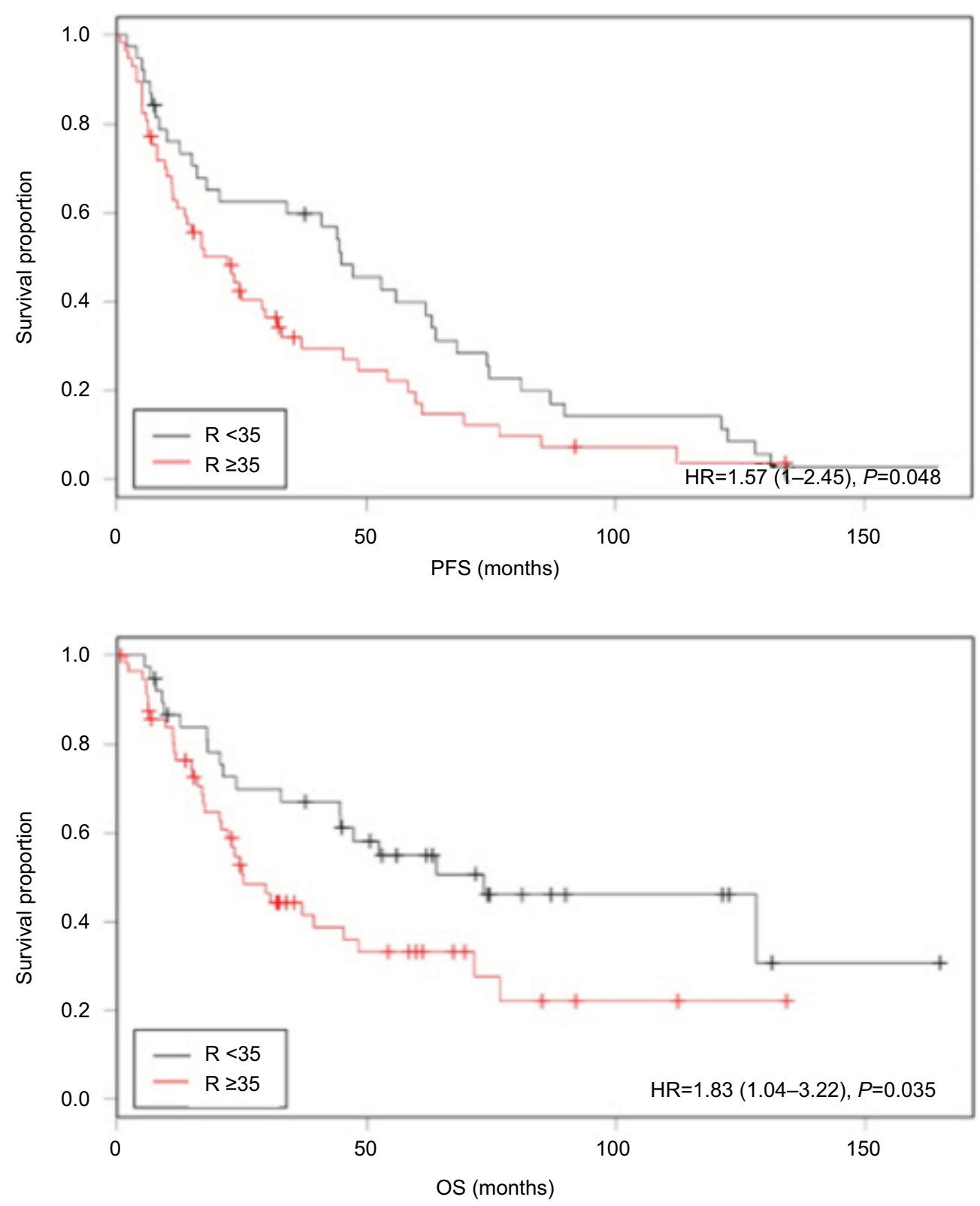

Figure 2 Kaplan-Meier (A) PFS and (B) OS curves in patients with SRC ratio $<35 \%$ vs $\geq 35 \%$.

Abbreviations: R, ratio; HR, hazard ratio; PFS, progression-free survival; OS, overall survival; SRC, signet ring cell.

adenocarcinoma in the same site ( 17 vs $34 \%, P<0.05) .{ }^{1}$ In addition, Sung et $\mathrm{al}^{9}$ and Tan et $\mathrm{al}^{13}$ demonstrated that colorectal MAC patients with SRCs show poorer outcome than those without. Moreover, SRCC is considered an independent predictive factor of dismal prognosis. ${ }^{14,15}$ Reportedly, the 5 -year OS rate of colorectal SRCC ranges from 0 to $31 \%$, ${ }^{5,13,15-17}$ obviously lower than that of the conventional colorectal MAC (range 48.0-69.0\%). ${ }^{9,13,17}$ In the current analysis, the 5 -year OS rate in colorectal MAC-SRC patients was $42.5 \%$, falling between MAC and SRCC rates. Interestingly, a previous study revealed that the morphological and molecular characteristics of colorectal MAC with low amounts of
SRCs $(<50 \%)$ are similar to those of SRCC..$^{18}$ Therefore, we hypothesized that the SRC ratio is associated with the dismal prognosis of colorectal MAC-SRC. As shown above, $35 \%$ was the cutoff point of the SRC ratio. The above results showed that the 5-year PFS and OS rates in patients with SRC ratios $<35 \%$ were higher than those of individuals with $\geq 35 \%$ (PFS, 37.0 vs $14.7 \%, P=0.048$; OS, 50.7 vs $33.2 \%$, $P=0.035$, respectively). Importantly, the SRC ratio was an independent prognostic factor of PFS $(P=0.014, \mathrm{HR}=1.917$, 95\% CI $1.140-3.225)$ and $\mathrm{OS}(P=0.032, \mathrm{HR}=2.500,95 \%$ CI 1.082-5.781). In general, refining the impact of the SRC ratio on survival could help implement more aggressive 
treatments for patients with poor prognosis, and provides a basis for building an optimal prognostic system. Therefore, pathologists are advised to identify any SRC component and focus intensively on patients with colorectal MAC-SRC, especially those with low amounts of SRCs. Based on our findings, 35\% might be an optimal cutoff point for predicting the outcome of colorectal MAC-SRC.

In agreement with previous colorectal SRCC data, ${ }^{4,5,7-9}$ colorectal MAC-SRC patients were commonly diagnosed at an advanced stage in this study, with distant metastasis or lymph node metastasis. In a previous study examining 59 patients, 58 (98.3\%) individuals had T3/T4 lesions, 52 (88.1\%) showed lymph node metastasis, and 14 (23.7\%) had distant metastasis. ${ }^{5}$ In line with previous reports, almost all cases $(96.3 \%)$ in the current study were diagnosed as stage T3 or T4, with $91.4 \%$ and $29.3 \%$ showing lymph node involvement and distant metastasis, respectively. Early diagnosis might be challenging because colorectal MAC-SRC is merely asymptomatic in the initial stage. However, SRCs are histologically more aggressive due to the absence of cellto-cell adhesion, which leads to a high tendency of metastasis. ${ }^{7,9,19}$ The reported incidence of peritoneal metastasis in colorectal SRCC is $38 \%$, with a combined rate at other sites, including the liver, lung, bone, and brain, of only $18.5 \%{ }^{4}$ Additionally, colorectal tumors with $<50 \%$ SRCs have a predisposition $(75.0 \%)$ to metastasize to the peritoneum and ovaries. ${ }^{19}$ Similarly, we observed that colorectal MAC-SRC metastasized predominantly to the peritoneum (40.7\%) and ovaries (29.6\%), and rarely to the liver, lung, and bone $(18.5 \%$ combined). For the 38 patients with $<35 \%$ SRCs, the rate of peritoneal and ovarian metastasis was $18.4 \%$, while $21.1 \%$ was obtained for the 57 patients with $\geq 35 \%$ SRCs. Hence, a high peritoneal and ovarian metastasis rate and a relatively low incidence of liver or lung metastasis may constitute a unique characteristic of colorectal MAC-SRC. This might be ascribed to poor prognosis since the patients cannot be treated by radical surgery to diffuse the peritoneal metastasis. In the current study, distant metastasis was an independent predictor of PFS $(P=0.044, \mathrm{HR}=1.750,95 \%$ CI 1.015-3.017) and OS $(P=0.000, \mathrm{HR}=3.219,95 \% \mathrm{CI} 1.676-6.181)$, indicating the poor outcome of MAC-SRC.

Previous studies demonstrated that colorectal SRCC is more common in younger individuals ( $\leq 40$-year-old) than non-SRC MAC. ${ }^{4,5,7,14}$ The reported mean age of colorectal SRCC patients is 48.1 years, while 57.4 years was found for colorectal MAC. ${ }^{20}$ Young age ( $\leq 40$-year-old) was shown to be an independent predictive factor of disease outcome in a previous study. ${ }^{7}$ In this work, the median age of colorectal MAC-SRC patients was 44 years, corroborating previous reports. However, no significant association was found between age and PFS $(P=0.871)$ or $\mathrm{OS}(P=0.959)$. This might be explained by the fact that all colorectal MAC-SRC patients were enrolled in this analysis, including those with reduced SRC amounts $(<50 \%)$.

$\mathrm{Fu}$ et $\mathrm{al}^{14}$ and Inamura et $\mathrm{al}^{15}$ reported that a female predominance is common (range 56-73.4\%), with females having a poor outcome in colorectal SRCC $(P<0.001),{ }^{14}$ while males show prevalence in other studies (range 64.4-68.2\%). ${ }^{4,5,20}$ Meanwhile, others found no significant difference between males and females. ${ }^{5,17}$ In the current work, females and males had equal rates ( 49.5 vs $50.5 \%$ ), with no significant association between gender and PFS $(P=0.199)$ or OS $(P=0.128)$, corroborating the findings of Wang et $\mathrm{al}^{5}$ and Hugen et al. ${ }^{17}$

Studies also showed a right-side predominance (44.0\%) in colorectal SRCC, ${ }^{21}$ while others consider the colonic site an independent prognostic factor $(P=0.012) .{ }^{14}$ As shown above, all sites were involved in colorectal MAC-SRC, with rectal MAC-SRC being rather common (62.1\%), in line with the literature. ${ }^{4,5}$ Moreover, Tawadros et $\mathrm{al}^{7}$ demonstrated that rectal cancer morbidity in young patients $(<40)$ increases with an annual change of $+3.6 \%$; in addition, cancers in this population are more likely to exhibit the SRC component (about 3.6:1). In this study, young patients with rectal MACSRC ( $\leq 40$-year-old) had a 5 -year OS rate of $40.2 \%$, while the median SRC ratio was $55 \%$.

The 8th AJCC Cancer Staging recommends pre-CEA and LVI as prognostic and predictive factors of colorectal cancer. ${ }^{11}$ Indeed, pre-CEA is considered an independent prognostic factor of colorectal cancer. ${ }^{22}$ In the current cohort, pre-CEA had a significant impact on OS as assessed by univariate analysis (5-year OS, 38.4 vs 42.0\%); however, it was not an independent predictive factor of MAC-SRC $(P=0.100)$. Meanwhile, LVI is considered an independent prognostic factor of SRCC $\left(\mathrm{HR}=2.888,95 \%\right.$ CI 1.115-7.483). ${ }^{5}$ In agreement, Barresi et $\mathrm{al}^{23}$ showed that VI, assessed by IHC staining, is an independent prognostic factor of disease-free survival $(P=0.02)$ and cancer-specific survival $(P=0.001)$. In the current cohort, LVI had a significant impact on PFS $(P=0.032)$ but was not an independent predictive factor of MAC-SRC $(P=0.112)$. The pathological characteristics were assessed by H\&E rather than IHC staining in this work, and we could not distinguish VI from LVI. Thus, IHC staining should be applied in further studies with larger sample sizes.

To the best of our knowledge, surgical resection is the gold standard treatment for resectable colorectal cancer. Nevertheless, SRCC cases display greater intolerability to surgery than non-SRCC ones, with recurrence or death 
within a short period of time post-surgery (24.5 vs $13.0 \%$, $P=0.001) .{ }^{14}$ Here, we observed that pre-CT and pre-RT could improve survival in colorectal MAC-SRC, unlike post-CT and post-RT. In contrast, a large population-based study in the Netherlands observed longer survival in stage III SRCC patients treated with post-CT; however, a significant interaction between SRCC and chemotherapy was not observed $(P=0.54) .{ }^{17}$ In addition, neither pre-RT nor post-RT was found to affect survival in SRCC. ${ }^{4}$ Due to its rarity, studies exploring the optimal treatment strategy for colorectal SRCC are scarce. However, because of the aggressive nature of SRC, it is necessary to gain insights into the potential treatment options to enhance survival in these patients.

\section{Limitations}

This retrospective study had some limitations. First, it was a single-center clinical research that only enrolled 95 patients with colorectal MAC-SRC due to the rarity of the disease. Thus, multicenter studies are warranted for further exploring the disease. Secondly, 14 patients underwent no surgery and $>10 \%$ had missing $\mathrm{T}$ - and $\mathrm{N}$-stage data in this retrospective analysis, which might result in selection bias. Thirdly, we provided only limited novel information about the morphological and molecular characteristics of SRCs, which necessitate additional studies.

\section{Conclusion}

Overall, we assessed the long-term survival outcomes and identified the SRC ratio as an independent prognostic factor of colorectal MAC-SRC. Moreover, M stage might be negatively correlated with prognosis, while pre-CT and preRT could prolong survival independently. However, further studies are required to confirm these findings and explore the molecular mechanisms underlying the SRC components that affect survival.

\section{Abbreviations}

AJCC, American Joint Committee on Cancer; CI, confidence interval; ESMO, European Society for Medical Oncology; H\&E, hematoxylin and eosin; HR, hazard ratio; IHC, immunohistochemical; LVI, lymphovascular invasion; MAC, mucinous adenocarcinoma; MAC-SRC, MAC with SRCs; OS, overall survival; PFS, progression-free survival; Post-CT, postoperative chemotherapy; Post-RT, postoperative radiotherapy; Pre-CEA, preoperative carcinoembryonic antigen; Pre-CT, preoperative chemotherapy; Pre-RT, preoperative radiotherapy; SRC, signet ring cell; SRCC, signet ring cell carcinoma; VI, vascular invasion

\section{Acknowledgments}

The authors would like to express their sincere gratitude to Yanping Chen and Xuehong Liao, for help with pathological diagnosis in this study.

This study was supported by the Fujian Province Natural Science Foundation (numbers 2016J01437 and 2017J01260), the Fujian Medical Innovation Project (number 2015-CX-8), the Peking University Cancer Hospital \& Institute, Key Laboratory of Carcinogenesis and Translational Research, Ministry of Education/Beijing (2017 Open Project-9), the Key Clinical Specialty Discipline Construction Program of Fujian, People's Republic of China, and the National Clinical Key Specialty Construction Program.

This work was presented as an abstract at the ESMO Congress, held in Madrid in September 2017 (abstract 499P).

\section{Disclosure}

The authors report no conflicts of interest in this work.

\section{References}

1. Secco GB, Fardelli R, Campora E, et al. Primary mucinous adenocarcinomas and signet-ring cell carcinomas of colon and rectum. Oncology. 1994;51(1):30-34.

2. el-Zimaity HM, Itani K, Graham DY. Early diagnosis of signet ring cell carcinoma of the stomach: role of the Genta stain. J Clin Pathol. 1997;50(10):867-868.

3. Verhulst J, Ferdinande L, Demetter P, Ceelen W. Mucinous subtype as prognostic factor in colorectal cancer: a systematic review and metaanalysis. J Clin Pathol. 2012;65(5):381-388.

4. Belli S, Aytac HO, Karagulle E, Yabanoglu H, Kayaselcuk F, Yildirim S Outcomes of surgical treatment of primary signet ring cell carcinoma of the colon and rectum: 22 cases reviewed with literature. Int Surg. 2014;99(6):691-698.

5. Wang R, Ma X, Li Y, et al. The characteristics and prognostic effect of E-cadherin expression in colorectal signet ring cell carcinoma. PLoS One. 2016;11(8): $\mathrm{e} 0160527$.

6. Almagro UA. Primary signet-ring carcinoma of the colon. Cancer. 1983;52(8):1453-1457.

7. Tawadros PS, Paquette IM, Hanly AM, Mellgren AF, Rothenberger DA, Madoff RD. Adenocarcinoma of the rectum in patients under age 40 is increasing: impact of signet-ring cell histology. Dis Colon Rectum. 2015;58(5):474-478.

8. Thota R, Fang X, Subbiah S. Clinicopathological features and survival outcomes of primary signet ring cell and mucinous adenocarcinoma of colon: retrospective analysis of VACCR database. J Gastrointest Oncol. 2014;5(1):18-24.

9. Sung CO, Seo JW, Kim KM, Do IG, Kim SW, Park CK. Clinical significance of signet-ring cells in colorectal mucinous adenocarcinoma Mod Pathol. 2008;21(12):1533-1541.

10. Bosman F, Carneiro F, Hruban R, Theise N. WHO Classification of Tumours of the Digestive System. 4th ed. Lyon: IARC; 2010.

11. Yao $\mathrm{H}, \mathrm{Wu} \mathrm{H}$, Liu Y. [Improvement of prognostic and predictive network of colorectal cancer based upon the 8th edition of AJCC colorectal cancer staging system]. Zhonghua Wei Chang Wai Ke Za Zhi. 2017;20(1):24-27. Chinese.

12. Budczies J, Klauschen F, Sinn BV, et al. Cutoff Finder: a comprehensive and straightforward Web application enabling rapid biomarker cutoff optimization. PLoS One. 2012;7(12):e51862. 
13. Tan Y, Fu J, Li X, et al. A minor ( $<50 \%)$ signet-ring cell component associated with poor prognosis in colorectal cancer patients: a 26-year retrospective study in China. PLoS One. 2015;10(3):e0121944.

14. Fu J, Wu L, Jiang M, et al. Signet ring cell carcinoma of resectable metastatic colorectal cancer has rare surgical value. J Surg Oncol. 2016;114(8):1004-1008.

15. Inamura $\mathrm{K}$, Yamauchi $\mathrm{M}$, Nishihara R, et al. Prognostic significance and molecular features of signet-ring cell and mucinous components in colorectal carcinoma. Ann Surg Oncol. 2015;22(4):1226-1235.

16. Lee HS, Soh JS, Lee S, et al. Clinical features and prognosis of resectable primary colorectal signet-ring cell carcinoma. Intest Res. 2015;13(4):332-338.

17. Hugen N, Verhoeven RH, Lemmens VE, et al. Colorectal signet-ring cell carcinoma: benefit from adjuvant chemotherapy but a poor prognostic factor. Int J Cancer. 2015;136(2):333-339.
18. Ogino S, Brahmandam M, Cantor M, et al. Distinct molecular features of colorectal carcinoma with signet ring cell component and colorectal carcinoma with mucinous component. Mod Pathol. 2006;19(1):59-68.

19. Pande R, Sunga A, Levea C, et al. Significance of signet-ring cells in patients with colorectal cancer. Dis Colon Rectum. 2008;51(1):50-55.

20. Chen JS, Hsieh PS, Hung SY, et al. Clinical significance of signet ring cell rectal carcinoma. Int J Colorectal Dis. 2004;19(2):102-107.

21. Ooi BS, Ho YH, Eu KW, Seow Choen F. Primary colorectal signet-ring cell carcinoma in Singapore. ANZ J Surg. 2001;71(12):703-706.

22. Li Destri G, Rubino AS, Latino R, et al. Preoperative carcinoembryonic antigen and prognosis of colorectal cancer. An independent prognostic factor still reliable. Int Surg. 2015;100(4):617-625.

23. Barresi V, Reggiani Bonetti L, Domati F, Baron L. Prognostic relevance of histopathological features in signet ring cell carcinoma of the colorectum. Virchows Arch. 2016;469(3):267-275.
Cancer Management and Research

\section{Publish your work in this journal}

Cancer Management and Research is an international, peer-reviewed open access journal focusing on cancer research and the optimal use of preventative and integrated treatment interventions to achieve improved outcomes, enhanced survival and quality of life for the cancer patient. The manuscript management system is completely online and includes

\section{Dovepress}

a very quick and fair peer-review system, which is all easy to use. Visit http://www.dovepress.com/testimonials.php to read real quotes from published authors. 